Pacific

Journal of

Mathematics

QUOTIENTS OF NILALGEBRAS AND

THEIR ASSOCIATED GROUPS

LAKHDAR HAMMOUDI

Volume 212 No. 1

November 2003 


\title{
QUOTIENTS OF NILALGEBRAS AND THEIR ASSOCIATED GROUPS
}

\author{
LAKHDAR HAMMOUDI
}

\begin{abstract}
We show that every finitely generated nilalgebra having nilalgebras of matrices is a homomorphic image of nilalgebras constructed by the Golod method (Golod, 1965 and 1969). By applying some elements of module theory to these results, we construct over any field non-residually finite nilalgebras and Golod groups with non-residually finite quotients. This solves Šunkov's problem (Kourovka Notebook, 1995, Problem 12.102). Also, we reduce Kaplansky's problem on the existence of a f.g. infinite $p$-group $G$ such that the augmentation ideal $\omega K[G]$ over a nondenumerable field $K$ is a nilideal (Kaplansky, 1957, Problem 9) to the study of the just-infinite quotients of Golod groups.
\end{abstract}

\section{Introduction.}

This paper deals with finitely generated (f.g.) infinite dimensional nilalgebras and their associated groups. Using Golod's algebras Anan'in and Puczyłowski constructed over fields of characteristic zero f.g. non-nilpotent nilalgebras which are not residually finite $[\mathbf{2}, \mathbf{1 5}]$. On the other hand, Rowen has proved their existence over every field [16]. Here we shall construct such examples over every field. This will enable us to solve in the negative Šunkov's problem [11, Problem 12.102] by constructing Golod groups with non-residually finite quotients. To this end we shall first start constructing Golod algebras as extensions of some nilalgebras. This is a completely different view from the classical one where Golod algebras are seen as homomorphic images. On the other hand the proofs of Theorems 2 and 3 are careful analysis of the Golod method. However, a great deal of information is extracted. For example, we prove that every f.g. nilalgebra over a nondenumerable field is a homomorphic image of a Golod algebra. As a consequence, Kaplansky's problem on the existence of a f.g. infinite $p$-group $G$ such that the augmentation ideal $\omega K[G]$ over a nondenumerable field $K$ is a nilideal [10, Problem 9] is reduced to the study of the just-infinite quotients of Golod groups. In the denumerable case we obtain some results, although because of the Köthe conjecture [12] the situation is quite complicated and we are far from understanding it. 
Let $K$ be any field and let $F^{(1)}$ be the free associative algebra of polynomials without constant terms in the non-commuting indeterminates $X_{1}, \ldots, X_{d}$ $(d \geq 2)$ over $K$. In this work an algebra means an associative algebra unless otherwise stated.

Lemma 1 ([6, 7]). Let I be an ideal of $F^{(1)}$ generated by a family of homogeneous polynomials $f_{1}, f_{2}, \ldots$ of non-decreasing degrees greater than or equal to 2. Let $r_{i}$ be the number of polynomials of each degree $i \geq 2$ in the sequence $f_{1}, f_{2}, \ldots$ If the coefficients of the series $\left(1-d t+\sum_{i=2}^{+\infty} r_{i} t^{i}\right)^{-1}$ are positive, then the algebra $F^{(1)} / I$ is of infinite dimension. In particular this is true if for a fixed real $\epsilon, 0<\epsilon<1 / 2, r_{i} \leq \epsilon^{2}(d-2 \epsilon)^{i-2}$, for every $i \geq 2$.

A Golod algebra is a f.g. non-nilpotent nilalgebra which satisfies Lemma 1 and which is constructed by the Golod method as in $[6,7]$.

An algebra $A$ over a field $k$ is absolutely nil if for every extension field $K \supset k, A \otimes K$ is a nilalgebra $\left[\mathbf{1}, 1_{c}\right.$, p. 51$]$.

We shall use the following characterization of absolutely nilalgebras:

Lemma 2 ([1, $3_{c}$, p. 52]). The algebra $A$ is absolutely nil if for every finite set $g_{1}, \ldots, g_{n}$ of elements of $A$, there exists an integer $m$ such that for every partition $m=\mu_{1}+\cdots+\mu_{n}, \mu_{i} \geq 0, \phi_{\mu_{1} \ldots \mu_{n}}\left(g_{1}, \ldots, g_{n}\right)=\sum g_{i_{1}} \cdots g_{i_{m}}=0$, where $\sum$ ranges over all the products which contain $g_{j}, \mu_{j}$ times for every $j$.

The smallest such integer $m$ is called the degree of absolute nillity of $g_{1}, \ldots, g_{n}$. It is obvious that $\phi_{\mu_{1} \ldots \mu_{n}}\left(g_{1}, \ldots, g_{n}\right)$ is a homogeneous polynomial of degree $m$ in the subalgebra generated by $g_{1}, \ldots, g_{n} . \phi_{\mu_{1} \ldots \mu_{n}}\left(g_{1}, \ldots\right.$, $\left.g_{n}\right)$ is called a $\phi_{\mu_{1}, \mu_{n}}\left(g_{1}, \ldots, g_{n}\right)$ homogeneous polynomial. When there is no ambiguity, we speak about the $\phi_{\mu_{1}, \mu_{n}}$ homogeneous polynomials (parts, components) where $\mu_{1}, \ldots, \mu_{n}$ range over all the partitions of $m$.

It is well-known that every f.g. nilalgebra over a nondenumerable field and every locally nilpotent algebra are absolutely nil [1]. It is observed [1, p. 56] and is proved below (see Remark 2) that Golod algebras are examples of non-locally nilpotent absolutely nilalgebras.

\section{Residually finite case.}

Theorem 1. Let $A=F^{(1)} / I$ be a nilalgebra with an absolutely nil ideal $J / I$ such that $J$ is a homogeneous ideal of $F^{(1)}$. Then $A$ is a homomorphic image of a residually finite nilalgebra $B=F^{(1)} / T$ such that $T$ is a homogeneous ideal. 
Proof. Let $g \in F^{(1)}$ and $n$ be an integer such that $g^{n} \in J$. Then $g^{n}=$ $\sum_{i} \mathcal{M}_{i}$, where $\mathcal{M}_{j}$ are homogeneous polynomials of $J$. Since $J / I$ is an absolutely nilalgebra, there exists an integer $m=m\left(\mathcal{M}_{i_{1}}, \ldots, \mathcal{M}_{i_{k}}\right)$ such that all the homogeneous polynomials in the $\mathcal{M}_{j}, \phi_{\mu_{1}, \mu_{k}}=\sum \mathcal{M}_{j_{1}} \cdots \mathcal{M}_{j_{m}} \in I$. But every element $\mathcal{M}_{j}$ is homogeneous in $F^{(1)}$, so all the polynomials $\phi_{\mu_{1}, \mu_{k}}$ are homogeneous in $F^{(1)}$. From the fact that $\left(g^{n}\right)^{m}=\sum_{\mu_{1}+\cdots+\mu_{k}=m} \phi_{\mu_{1}, \mu_{k}}$ we see that $\left(g^{n}\right)^{m}$ is a sum of homogeneous elements of $I$. Let $T$ be the ideal of $F^{(1)}$ generated by all the homogeneous polynomials $\phi_{\mu_{1}, \mu_{k}}$, so constructed. It is obvious that $T \subset I$ is a homogeneous ideal and that $F^{(1)} / T$ is a residually finite nilalgebra.

In view of this theorem we ask the following natural question:

Question 1. Let $A$ be an algebra as in the previous theorem. Is $A$ absolutely nil?

Although this question seems to be difficult, one can observe that if $J / I$ is an ideal of $A$ of finite codimension then $A$ is absolutely nil. This gives the following characterization of f.g. non-absolutely nilalgebras. Examples of this sort are the nilalgebras generated by 3 elements constructed recently by Smoktunowicz [18].

Corollary 1. Let $A$ be a f.g. non-absolutely nilalgebra. Then for every $n \geq$ $1, A^{n}$ is a f.g. nilalgebra which is not absolutely nil.

Theorem 2. Let $A=F^{(1)} / I$ be a nilalgebra over a denumerable field such that $I$ is a homogeneous ideal. Then $A$ is a homomorphic image of a residually finite nilalgebra $B=F^{(1)} / J$ which satisfies Lemma 1 .

Proof. We will construct by induction a family of homogeneous polynomials $f_{1}, f_{2}, \ldots$ which generate the ideal $J$.

We suppose that the base field $K$ is denumerable. In this case $F^{(1)}$ is denumerable. Let us enumerate its elements as $\left\{y_{1}, y_{2}, \ldots\right\}$. Choose an integer $n$ greater than or equal to the index of nilpotency of $\left(y_{1}+I\right)$. Then $y_{1}^{n}$ is in $I$ and since $I$ is homogeneous, each of its homogeneous components $f_{1}, \ldots, f_{t}$ (with $\operatorname{deg} f_{j}<\operatorname{deg} f_{j+1}$ ) is in $I$. Given any number $k$, there is no more than one $f_{i}$ with degree $k$. So we have the set $\left\{f_{1}, \ldots, f_{t}\right\}$ satisfying Lemma 1. In particular, there exit homogeneous polynomials $f_{1}, \ldots, f_{t}$ with increasing degrees in $I$ satisfying Lemma 1 such that $y_{1}^{n}$ is in the ideal generated by $\left\{f_{1}, \ldots, f_{t}\right\} \subseteq I$.

Suppose by induction, we have a Golod set $\left\{f_{1}, \ldots, f_{s}\right\} \subseteq I$ such that $\operatorname{deg} f_{i}<\operatorname{deg} f_{i+1}$ and for each $i=1, \ldots, k$ there is an integer $n_{i}$ with $y_{i}^{n_{i}}$ in the ideal generated by $\left\{f_{1}, \ldots, f_{s}\right\}$. For $y_{k+1}$ choose an integer $m$ greater than both the index of nilpotency of $\left(y_{k+1}+I\right)$ and $\operatorname{deg} f_{s}$. Since $A$ is 
nil and since $I$ is a homogeneous ideal, we can write $\left(y_{k+1}\right)^{m}$ in terms of its homogeneous components all of which are in $I$, and all of which have degree larger than $\operatorname{deg} f_{s}$. Label these components $f_{s+1}, \ldots, f_{r}$. Then the set $\left\{f_{1}, \ldots, f_{s}, f_{s+1}, \ldots, f_{r}\right\} \subseteq I$ of homogeneous polynomials satisfies Lemma 1 such that for each $i=1, \ldots, k+1$, there is an integer $n_{i}$ with $y_{i}^{n_{i}}$ in the ideal generated by $\left\{f_{1}, \ldots, f_{r}\right\}$. Now, by the induction we have an infinite set of homogeneous polynomials $f_{1}, f_{2}, \ldots$ in $I$ satisfying Lemma 1 , and which generates the ideal $J$, such that $F^{(1)} / J$ is a nilalgebra.

Theorem 3. Let $A=F^{(1)} / I$ be an absolutely nilalgebra. Then $A$ is a homomorphic image of a Golod algebra $B=F^{(1)} / J$.

Proof. The proof is by induction on the degrees of general polynomials. Let $g_{1}=c_{1} X_{1}+\cdots+c_{d} X_{d}$ be a general polynomial of degree 1 in $F^{(1)}$ and choose an integer $l$ greater than or equal to the degree of absolute nillity of $X_{1}+I, \ldots, X_{d}+I$. Since $A$ is absolutely nil, by Lemma 2, for every partition $l=\mu_{1}+\cdots+\mu_{d}, \mu_{i} \geq 0$, the $\phi_{\mu_{1}, \mu_{d}}\left(X_{1}, \ldots, X_{d}\right)$ polynomials are in $I$. These polynomials are just the coefficients (homogeneous polynomials in $X_{1}, \ldots, X_{d}$ ) of $g_{1}^{l}$ when seen as a polynomial in the commuting unknowns $c_{1}, \ldots, c_{d}$. Let us denote these $\phi_{\mu_{1}, \mu_{d}}\left(X_{1}, \ldots, X_{d}\right)$ polynomials as $f_{1}, \ldots, f_{l_{1}}$. Now, since the number $r_{i}$ of polynomials of each degree $i$ (in this case $i=l$ ) in $\left\{f_{1}, \ldots, f_{l_{1}}\right\}$ does not exceed $(l+d-1)^{d-1}$, for $l$ big enough, $r_{i} \leq(l+$ $d-1)^{d-1} \leq \epsilon^{2}(d-2 \epsilon)^{i-2}$. Thus, the set $\left\{f_{1}, \ldots, f_{l_{1}}\right\}$ satisfies Lemma 1 .

Suppose that we have constructed in $I$ a system of homogeneous polynomials $f_{1}, \ldots, f_{l_{k}}$ satisfying Lemma 1 and that for every polynomial $y \in F^{(1)}$ of a degree not exceeding $k$ there exists an integer $l^{\prime}=l^{\prime}(y)$ such that the homogeneous parts of $y^{l^{\prime}}$ are in the ideal generated by $f_{1}, \ldots, f_{l_{k}}$. Let

$$
\begin{aligned}
g_{k+1}=c_{1}^{(1)} X_{1}+\cdots+c_{d}^{(1)} X_{d}+c_{1}^{(2)} X_{1}^{2}+c_{2}^{(2)} X_{1} X_{2}+\cdots+ & \\
& c_{d^{2}}^{(2)} X_{d}^{2}+\cdots+c_{d^{k+1}}^{(k+1)} X_{d}^{k+1}
\end{aligned}
$$

be a general polynomial of $F^{(1)}$ of degree $k+1$. Let $n$ be an integer greater than $\max \left(\operatorname{deg} f_{1}, \ldots, \operatorname{deg} f_{l_{k}}, m\left(X_{1}, \ldots, X_{1} X_{d}, \ldots, X_{d}^{k+1}\right)\right)$, where, $m\left(X_{1}\right.$, $\left.\ldots, X_{d}^{k+1}\right)$ is the degree of absolute nillity of $X_{1}, \ldots, X_{1} X_{d}, \ldots, X_{d}^{k+1}$. By Lemma 2, for every partition $n=\mu_{1}+\cdots+\mu_{q}, \mu_{i} \geq 0, q=d+\cdots+d^{k+1}$ the $\phi_{\mu_{1}, \mu_{q}}\left(X_{1}, \ldots, X_{d}^{k+1}\right)$ polynomials are in $I$. As in the case of $g_{1}$, by the choice of the integer $n$, the coefficients of $g_{k+1}^{n}$, seen as a polynomial in the commuting unknowns $c_{1}^{(1)}, \ldots, c_{d^{k+1}}^{(k+1)}$, are the $\phi_{\mu_{1}, \mu_{q}}\left(X_{1}, \ldots, X_{d}^{k+1}\right) \in I$. Let us denote them by $f_{l_{k}+1}, \ldots, f_{l_{k+1}}$ and construct a new family of homogeneous polynomials $f_{1}, \ldots, f_{l_{k}}, f_{l_{k}+1}, \ldots, f_{l_{k+1}}$ satisfying Lemma 1 . Indeed, the number $r_{i}$ of polynomials of degree $i>\max \left(\operatorname{deg} f_{1}, \ldots, \operatorname{deg} f_{l_{k}}\right)$ does not exceed $(n+q-1)^{q-1}$. For $n$ big enough, we have $r_{i} \leq(n+q-1)^{q-1} \leq$ $\epsilon^{2}(d-2 \epsilon)^{i-2}$. For $i \leq \max \left(\operatorname{deg} f_{1}, \ldots, \operatorname{deg} f_{l_{k}}\right)$ this property is satisfied in 
the system $f_{1}, \ldots, f_{l_{k}}$. So we have constructed a family of polynomials $f_{1}, \ldots, f_{l_{k+1}}$ satisfying Lemma 1 and for every polynomial $z \in F^{(1)}$ of a degree not exceeding $k+1$ there exists an integer $n^{\prime}=n^{\prime}(z)$ such that the homogeneous parts of $z^{n^{\prime}}$ are in the ideal generated by $f_{1}, \ldots, f_{n_{k+1}}$. The union of all these families so constructed gives an infinite system of homogeneous polynomials $f_{1}, f_{2}, \ldots$ which generate the ideal $J$. We have proved the theorem.

\section{Remarks.}

1. If $A$ is such that specific elements generate a nilpotent (soluble, finite dimensional,...) subalgebra, then one can construct $B$ with the same properties as $A$.

2. From the proof of Theorem 3, we see that the Golod algebras are absolutely nil. Therefore, Golod algebras have nilalgebras of matrices. This solves P.M. Cohn's question [4, p. 387 and Exercise $6^{\circ}$, p. 395].

Having in mind that a f.g. nilalgebra over a nondenumerable field is absolutely nil [1], we obtain:

Corollary 2. Every f.g. nilalgebra over a nondenumerable field is a homomorphic image of a Golod algebra.

Let $A$ be a Golod algebra generated by $\bar{X}_{1}, \ldots, \bar{X}_{d}(d \geq 2)$. The group generated by $1+\bar{X}_{1}, \ldots, 1+\bar{X}_{d}$ is called the Golod group of $A$ and the Lie algebra generated by $\bar{X}_{1}, \ldots, \bar{X}_{d}$ is the Golod-Lie algebra.

Corollary 3. For any integer $d \geq 2$, every $d$-generator group arising from an absolutely nilalgebra is a homomorphic image of a d-generator Golod group. In particular, so is every finite p-group, for every prime integer $p$.

In [10, Problem 9], Kaplansky asked whether the augmentation ideal $\omega K[G]$ of a f.g. infinite $p$-group $G$ could be a nilideal. A particular case is Passman's question on the use of Golod groups to solve this problem $[\mathbf{1 3}$, p. 121 and Problem 18, p. 133], [14, p. 415]. The following result confirms Passman's observation and reduces Kaplansky's problem to the study of the quotients of Golod groups:

Corollary 4. Let $K$ be a nondenumerable field of characteristic $p>0$. Then, there exists a f.g. infinite p-group $\bar{G}$ such that the augmentation ideal $\omega K[\bar{G}]$ is nil if and only if there exists a just-infinite homomorphic image $G$ of a Golod p-group such that $\omega K[G]$ is nil.

Proof. Let $\bar{G}$ be as in the corollary. Since it is f.g and infinite., it has a just-infinite homomorphic image $G$. Hence, the augmentation ideal $\omega K[G]$ is a quotient of $\omega K[\bar{G}]$ and so it is a nilalgebra over a nondenumerable field $K$. By Corollary 2, $G$ and $\bar{G}$ are quotients of a Golod group. The converse is obvious. 
On the other hand we point out that since non-absolutely nilalgebras cannot be quotients of Golod algebras, their associated groups have non-nil augmentation ideals. The only examples of this type are the nilalgebras generated by 3 elements constructed by Smoktunowicz [18]. The following result is analogous to the results obtained in the case of the 2-generated Grigorchuk groups [5], the 3-generated Gupta-Sidki groups [17] and the free Burnside groups [9]:

Corollary 5. Let $K$ be a nondenumerable field of characteristic $p>0$. Let $G$ be a f.g. p-group associated to a non-absolutely nilalgebra. Then the augmentation ideal $\omega K[G]$ is not nil. Moreover $\omega K[G]$ has a just-infinite primitive homomorphic image.

Question 2. Could the group algebra in the preceeding Corollary contain a free associative algebra with two non-commuting indeterminates?

Corollary 6. For any integer $d \geq 2$, every d-generator Lie algebra arising from an absolutely nilalgebra is a homomorphic image of a d-generator Golod-Lie algebra.

\section{Non-residually finite case.}

We turn now to non-residually finite quotients of nilalgebras and their associated groups. We point out that a f.g. just-infinite nilalgebra or a f.g. just-infinite Jacobson radical ring is residually finite $[\mathbf{9}]$ and that some infinite dimensional quotients of Golod algebras are also Golod algebras (the same result holds for Golod groups and Golod-Lie algebras) [8, 19]. A subset $E$ of a ring $A$ is $T$-nilpotent if for every sequence $g_{1}, g_{2}, \ldots$ of elements of $E$, there exists an integer $k$ with $g_{1} g_{2} \cdots g_{k}=0$. It is obvious that $T$-nilpotency implies local nilpotency. In our investigations, a key role is played by the following generalization of Nakayama's lemma:

Lemma $3([\mathbf{2 0}, \S 43.5$, p. 386]). Let $A$ be an algebra. Then, $A M \neq M$ for every left $A$-module $M$, if and only if $A$ is T-nilpotent.

The existence of f.g. non-residually finite, infinite dimensional nilalgebras over every field was first proved in [16]. A simple observation yields a stronger result. Indeed, let $d \geq 2$ be an integer and suppose that for any $d$-generator nilalgebra $A$, any left $A$-module $M$ satisfies $\cap A^{i} M=\langle 0\rangle$. So, $A M \neq M$ and by Lemma $3, A$ is $T$-nilpotent. Thus every $d$-generator nilalgebra is nilpotent. This contradicts the Golod construction [6, 7] and proves:

Proposition. For every integer $d \geq 2$ and over any field, there exists a non-residually finite, non-nilpotent d-generator nilalgebra.

Theorem 4. Over any field, any f.g. non-nilpotent nilalgebra with involution is a homomorphic image of a f.g. non-residually finite nilalgebra. 
Proof. Let $A$ be a f.g. non-nilpotent nilalgebra with involution. Since $A$ is not locally finite, by Lemma 3 there exists a nondegenerate left $A$-module $M$ such that $A M=M$. It is well-known that every left $A$-module can be considered as a right module over the opposite algebra $A^{\circ}$ of $A$. But the fact that $A$ has an involution yields $A \cong A^{\circ}$ and turns $M$ to a nondegenerate $(A, A)$-bimodule such that $A M=M A=M$. Let $m$ be a nondegenerate element of $M$ and consider the submodule $N=\langle m\rangle$. Since $A$ has an involution and $N$ is nondegenerate, we have $A N=N A=N$. Denote by $\bar{A}$ the trivial extension of $A$ by $N$,

$$
\bar{A}=\{(a, n), a \in A, n \in N\} .
$$

With the usual addition and the following multiplication:

$$
(a, n)\left(a^{\prime}, n^{\prime}\right)=\left(a a^{\prime}, a n^{\prime}+n a^{\prime}\right), \quad a, a^{\prime} \in A, \quad n, n^{\prime} \in N,
$$

$\bar{A}$ is a non-nilpotent nilalgebra such that $\bar{A} / I=A$, where $I$ is the ideal $\langle(0, n), n \in N\rangle$. From the fact that $A N=N A=N$, it follows that $I$ is in $\bar{A}^{k}$ for every integer $k$; thus $\bar{A}$ is not residually finite. Since $A$ is f.g. and $N=\langle m\rangle, \bar{A}$ is f.g. Therefore, we proved the theorem.

Corollary 7. Over every field, there exists a Golod algebra with non-residually finite quotients.

Proof. Apply Theorems 1 and 2 or 3 to the non-residually finite nilalgebras of Theorem 4.

The following corollary solves in the negative Šunkov's problem $[\mathbf{1 1}$, Problem 12.102]:

Corollary 8. For every prime $p$ (respectively $p=0$ ), there exists Golod $p$ groups (respectively torsion free groups) with non-residually finite quotients.

Proof. Let $\bar{A}$ be a non-residually finite homomorphic image of a Golod algebra $B$ and denote by $Y_{1}, \ldots, Y_{d}$ its generators which are images of fixed generators of $B$. Since $\bar{A}$ is f.g., and $N=\langle m\rangle$ is a nondegenerate module satisfying $A N=N A=N$ (see the proof of Theorem 4), $1+(0, m) \in \bar{G}$ where, $\bar{G}=\left\langle 1+Y_{1}, \ldots, 1+Y_{d}\right\rangle$. Thus the Golod group of $B$ has $\bar{G}$ as a non-residually finite quotient.

We conclude with the following question which is related to Bergman's [3, Question 63]:

Question 3. Anan'in and Puczyłowski constructed over fields of characteristic zero, f.g. non-residually finite, non-nilpotent nilalgebras with nonradical tensor square $[\mathbf{2}, \mathbf{1 5}]$. Could we construct such examples in characteristic $p>0$ ? 


\section{References}

[1] S.A. Amitsur, Nil radicals. Historical notes and some new results, Colloq. Math. Soc. J. Bolyai, 6, Rings, Modules and Radicals, Bolyai J. Mat. Társulat, Budapest, 1973, 47-65, MR 50 \#374, Zbl 0263.16009.

[2] A.Z. Anan'in, A nil algebra with nonradical tensor square, Sibirsk. Mat. Zh., 26(2) (1985), 192-194, 224 (in Russian), MR 86i:16006, Zbl 0569.16017.

[3] G. Bergman, Radicals, tensor products, and algebraicity, Israel Math. Conf. Proc., 1, The Weizmann Science Press of Israel, 1989, 150-192, MR 91d:16033, Zbl 0674.16004.

[4] P.M. Cohn, Skew Fields. Theory of General Division Rings, Encyclopedia of Math. Appl., 57, Cambridge University Press, Cambridge, 1995, MR 97d:12003, Zbl 0840.16001.

[5] G. Genov and P.N. Siderov, Some properties of the Grigorchuk group and its group algebra over a field of characteristic two, Serdica, 15(4) (1989-1990), 309-326 (in Russian), MR 91h:20006, Zbl 0713.20029.

[6] E.S. Golod, On nilalgebras and finitely approximable p-groups, Amer. Math. Soc. Transl. Ser. 2, 48 (1965), 103-106, MR 28 \#5082, Zbl 0215.39202.

[7] _ Some problems of Burnside type, Amer. Math. Soc. Transl. Ser. 2, 84 (1969), 83-88, MR 39 \#240, Zbl 0206.32402.

[8] L. Hammoudi, Le nombre d'images homomorphes de certains groupes et algèbres de Golod, Results Math., 29(3-4) (1996), 227-232, MR 97i:16014, Zbl 0862.16017.

[9] _ Just-infinite nil-rings and groups, Algebra Colloq., 5(4) (1998) 371-376, MR 2000b:16035, Zbl 0940.20046.

[10] I. Kaplansky, Problems in the theory of rings, Report of a conference of linear algebra, NAS-NRC. Publ., 502, Washington, D.C., 1957, 1-3, MR 20 \#3179, Zbl 0095.25602.

[11] Kourovka Notebook, Unsolved problems in group theory, Novosibirsk, 1995.

[12] J. Krempa, Logical connections between some open problems concerning nil rings, Fund. Math., 76 (1972), 121-130, MR 46 \#5377, Zbl 0236.16004.

[13] D.S. Passman, Infinite Group Rings, Pure and Applied Mathematics, 6, Marcel Dekker, New York, 1971, MR 47 \#3500, Zbl 0221.20042.

[14] _ The Structure of Group Rings, Pure and Applied Mathematics, WileyInterscience, John Wiley \& Sons, New York, 1977, MR 81d:16001, Zbl 0368.16003.

[15] E.R. Puczyłowski, Nil-algebras with non-radical tensor square, Proc. Amer. Math. Soc., 103(2) (1988), 401-403, MR 89e:16021, Zbl 0661.16006.

[16] L.H. Rowen, Köthe's conjecture, Israel Math. Conf. Proc., 1, The Weizmann Science Press of Israel, 1989, 193-202, MR 90j:16073, Zbl 0688.16005.

[17] S.N. Sidki, A primitive ring associated to a Burnside 3-group, J. London Math. Soc., 55(1) (1997), 55-64, MR 97m:16006, Zbl 0874.20028.

[18] A. Smoktunowicz, Polynomial rings over nil rings need not be nil, J. Algebra, 233 (2000), 427-436, MR 2001i:16045, Zbl 0969.16006.

[19] A.V. Timofeenko, Infinite subgroups of infinite index in 2-generated p-groups of Golod type, Sibirsk. Mat. Zh., 27(5) (1986), 194-195 (in Russian), MR 88e:20040, Zbl 0612.20017.

[20] R. Wisbauer, Foundations of Module and Ring Theory. A Handbook for Study and Research, Revised and translated from the 1988 German edition, Algebra, Logic 
and Applications, 3, Gordon and Breach Science Publishers, Philadelphia, 1991, MR 92i:16001, Zbl 0746.16001.

Received April 20, 2001 and revised November 22, 2002.

Department of Mathematics

OHIO UNIVERSITY

571 West Fifth Street

Chillicothe, OH 45601

E-mail address: hammoudi@ohio.edu 